\title{
European Palliative Sedation Project
}

\author{
Sheila A. Payne, PhD, Editorial Board Member and Jeroen Hasselaar, PhD, Project Co-Ordinator
}

A S PATIENTS APPROACH the end of life, they often experience a range of distressing symptoms and concerns such as pain, delirium, and breathlessness. Although most symptoms and concerns are amendable to pharmacological and/or nonpharmacological interventions, there may be some symptoms that are very difficult or impossible to treat or where available treatment options fail. These are called refractory symptoms because treatment (1) does not work, (2) the effects take too long to happen, or (3) the side effects are not acceptable to the patient. Refractory symptoms are not restricted to physical symptoms and, therefore, a multidimensional approach is needed to fully assess and manage the patient's condition. For example, existential suffering may be adding to the patient's physical suffering but can be particularly difficult to assess and manage. For this reason, the term intractable suffering is sometimes used. When conventional treatment options are no longer available and symptoms are considered refractory, the option of palliative sedation comes to the fore.

Palliative sedation has been defined in different and sometimes even in conflicting ways, but as a core element it involves the intentional lowering of a patient's consciousness to relieve patient's discomfort. Precautionary conditions are that the patient is suffering from refractory symptoms and that the patient is in the last phase (one to two weeks) of life. The latter is important not only to avoid connotations of hidden euthanasia, but also to restrict this intervention to terminally ill patients. In these cases, the use of palliative sedation may vary in its duration (intermittent or continuous until death) and depth (light or deep). In all cases the intention of clinicians should be to provide relief of distressing symptoms, not to hasten death. Medication (mostly Midazolam) and dosages need to be titrated toward this goal of symptom relief.

Palliative sedation is estimated to precede $10 \%-18 \%$ of all deaths in Europe, although there is known to be considerable diversity in its use. Although there are national and international clinical guidelines for palliative sedation [such as the European Association for Palliative Care (EAPC) Framework $\left.^{1}\right]$, the quality and content of guidelines vary because the terminology and concepts lack consensus, and they are infrequently used. ${ }^{2-4}$ The practice of palliative sedation, in particular when it is applied as continuous deep sedation until death, raises important and controversial issues because some people have suggested that it may result in life-shortening effects and potentially result in what has been described as "slow" euthanasia, although the empirical evidence does not support this contention. Also, the use of palliative sedation for patients with mainly existential suffering remains controversial. ${ }^{5}$ The practice of palliative sedation, therefore, raises many practical and ethical dilemmas for clinicians and is often poorly understood by patients and families. Witnessing the "intolerable" suffering of others is distressing, both for family members and the clinical team. There is a lack of clarity in the assessment and management of refractory symptoms, to determine the right moment to start palliative sedation, the influence of different cultural, religious, and social norms, and how health professionals, patients, and families understand these (decision) processes and treatment options.

\section{What Is the Palliative Sedation Project About?}

Our new project, called Palliative Sedation, is funded by the Horizon 2020 program at the European Commission, for five years from January 2019. The project is led by Dr. Jeroen Hasselaar from Radboud University Medical Centre, Nijmegen, the Netherlands, along with eight other internationally renowned universities and clinical research centers. The project is also supported by the EAPC to ensure that patient and professional perspectives are addressed at all stages of the project. The International Observatory on End of Life Care at Lancaster University, United Kingdom, will prepare free online education that will ensure that clinicians and patients benefit from improvements arising from the project. For more information about the project, see our website www.palliativesedation.eu or follow the project on Twitter $@$ PallSed.

\section{How Will the Project Investigate Palliative Sedation?}

We aim to examine the concept and practice of proportional palliative sedation for refractory symptoms throughout Europe, where sedation is regarded on a continuum from light to deep, and where medication is titrated to that required for symptom relief to ensure maximum patient comfort and quality of dying.

International Observatory on End of Life Care, Division of Health Research, Faculty of Health and Medicine, Furness College, Lancaster University, Lancaster, United Kingdom.

(C) Sheila A. Payne and Jeroen Hasselaar 2019; Published by Mary Ann Liebert, Inc. This Open Access article is distributed under the terms of the Creative Commons Attribution Noncommercial License (http://creativecommons.org/licenses/by-nc/4.0/) which permits any noncommercial use, distribution, and reproduction in any medium, provided the original author(s) and the source are credited. 
Our project will use a number of research methods to investigate the assessment of refractory symptoms and the use of palliative sedation.

- Literature reviews: we start by examining the extensive published evidence to determine both its quality and its usefulness in defining terminology. For example, we are conducting an integrative literature review about the assessment and monitoring tools used to determine level and quality of palliative sedation.

- International country survey: we will investigate the degree of integration of clinical recommendations into current clinical practice in Europe and obtain an overview of national practices.

- Observational study of clinical practice in five countries (The Netherlands, Italy, Germany, Belgium, and Spain) to examine local practices and identify if and how palliative sedation, in its several subtypes, relates to better patient comfort using an observational study method (see also Van Deijck et al. ${ }^{6}$ ).

- Multiple case studies will provide in-depth information on patient cases in each clinical site, using qualitative methods.

- Moral case deliberations: we will use vignettes and "real cases" of palliative sedation to elicit discussions about end-of-life decision making with groups of clinicians in eight countries (The Netherlands, Italy, Germany, Belgium, Hungary, Romania, England, and Spain).

- Cost consequence analysis will be used to inform policy recommendations.

\section{What Are the Likely Benefits of This Research?}

This project is likely to provide important new evidence about how refractory symptoms are managed and how palliative sedation is used in Europe to increase patient comfort in the final days of life. We will start to understand more about clinical decision making near the end of life from the moral case deliberations, and identify differences and similarities in clinical practice across Europe, and the factors that influence them.

The project has some specific activities and outputs including the following:

- Revising and updating the EAPC Framework on Palliative Sedation (first published in 2009).

- Preparing and delivering a free online education program for clinicians and patients about what dying well means and how palliative sedation can be used to increase quality of dying with better symptom management. It will also highlight ethical and moral dilemmas for both health professionals and society.

- We will publish an ebook that will provide examples of how refractory symptoms are managed and illustrate how palliative sedation is used in different European countries. The book will highlight "best practices" and tools to guide clinicians facing these complex treatment challenges.
- Throughout the project, we will be presenting our findings at international conferences and in publications that will target different audiences, including health professionals, patients and family carers, researchers, and policy makers.

We have started down the path of research into a very complex and potentially controversial treatment option where ethical and clinical issues coincide. Since palliative sedation has become an increasingly common option in some countries for patients at end-of-life stages, it is essential that it should be critically examined with compassion and wisdom in cooperation with multiple experts, including patients and their families. We hope our project will provide the foundation to support global and national standards.

\section{Funding Information}

This project has received funding from the European Union's Horizon 2020 research and innovation programme under grant agreement No. 825700 .

\section{References}

1. Cherny NI, Radbruch L; Board of the European Association for Palliative Care: European Association for Palliative Care (EAPC) recommended framework for the use of sedation in palliative care. Palliat Med 2009;23:581-593.

2. Abarshi E, Rietjens J, Robijn L, et al.: International variations in Clinical Practice Guidelines for Palliative Sedation: A systematic review. BMJ Support Palliat Care 2017;7:223-229.

3. Abarshi E, Payne S: Awareness of the EAPC recommended framework for the use of sedation in palliative care: A webbased survey. Eur J Palliat Care 2014;21:81-85.

4. Papavasiliou E, Payne S, Brearley S; on behalf of EUROIMPACT: Current debates on end-of-life sedation: An international expert elicitation study. Support Care Cancer 2014:22;2141-2149.

5. Rodrigues P, Menten J, Gastmans C: Physicians' perceptions of palliative sedation for existential suffering: A systematic review. BMJ Support Palliat Care 2019. DOI:10.1136/ bmjspcare-2019-001865

6. van Deijck RH, Hasselaar JG, Verhagen SC, et al.: Level of discomfort decreases after the administration of continuous palliative sedation: A prospective multicenter study in hospices and palliative care units. J Pain Symptom Manage 2016;52:361-369.

Address correspondence to: Sheila A. Payne, PhD

Division of Health Research Faculty of Health and Medicine Furness College International Observatory on End of Life Care Lancaster University Lancaster LA1 4YG United Kingdom

E-mail: s.a.payne@lancaster.ac.uk 